\title{
LA IMPORTANCIA DE REALIZAR INVESTIGACIÓN EN CIENCIAS NATURALES EN EL NIVEL PREESCOLAR: LA BIOFILIA COMO UNA OPORTUNIDAD
}

\section{THE IMPORTANCE OF RESEARCHING IN SCIENCE IN PRESCHOOL LEVEL: THE BIOPHILIA AS AN OPPORTUNITY}

\author{
Hugo Torres-Contreras \\ Especialista en Ecología, Conducta y Evolución de Organismos Sociales \\ Departamento de Educación, Facultad de Ciencias Sociales, Universidad de Chile \\ Capitán Ignacio Carrera Pinto 1045, Ñuñoa, Santiago \\ htoresco@uchile.cl
}

Resumen: Existe escasa información sobre los aprendizajes de los preescolares chilenos en el ámbito de las Ciencias Naturales. Tomando en consideración la empatía innata que exhiben los niños pequeños por otros seres vivos, en este artículo se describen las habilidades de pensamiento científico observar y preguntar, y los cambios en nivel de complejidad que éstas experimentan a través del tiempo. Además, en el contexto de un proyecto FONDECYT Regular, se comentan las primeras actividades realizadas con niños del primer nivel de transición cuando éstos son confrontados a ejemplares de fauna nativa, y los registros de observaciones y preguntas que son capaces de efectuar a partir de dibujos y entrevistas. La dependencia administrativa del colegio, el nivel de vocabulario con que ingresan los niños, la interacción que establecen con sus padres en ambientes naturales, y la enseñanza que reciben de parte de sus educadoras de párvulos, son variables que podrían tener implicancias en las habilidades científicas de los niños.

Palabras clave: indagación científica, observación, pregunta, dibujo, fauna nativa

\begin{abstract}
There is scarce information about learning of Chilean preschoolers in science area. Considering the innate empathy exhibited by young children towards other living beings, this article describes the scientific thinking skills of observing and asking, and the evolution in complexity of these experiences through the time. Besides, in the context of a Grant, the first activities with preschoolers are discussed as they are confronted with native wildlife, and the record (drawings, interviews) of observations and questions that they are able to perform. The school type, level of initial vocabulary, interaction with their parents in natural environments, and teaching they receive from their educators, are variables that could have implications on scientific skills of children.
\end{abstract}

Keywords: scientific inquiry, observation, question, drawing, native wildlife 


\section{INTRODUCCIÓN}

Existe escasa información publicada en revistas indexadas (ISI, Scielo, Scopus) sobre investigaciones científicas que aborden los temas de aprendizaje en el nivel de la Educación Parvularia en Chile (López de Maturana, 2010; Hepp et al., 2013; Sánchez, 2014). En particular, hasta la fecha no existen antecedentes sobre los aprendizajes que experimentan los preescolares en el ámbito de las Ciencias Naturales. Este hecho llama especialmente la atención, dado que el nivel de Educación Parvularia constituye la puerta de entrada al sistema escolar chileno, y a pesar de esto no se han documentado, entre otros, los cambios que experimentan los niños en sus habilidades de pensamiento científico durante este período. Las evidencias que se encuentran luego de efectuar búsquedas bibliográficas, son algunas aproximaciones indirectas a esta problemática como los aportes efectuados por investigadores nacionales en el ámbito de la didáctica de las ciencias naturales (Daza y Quintanilla, 2011), la formación en ciencias por parte de las educadoras de párvulos y su impacto en el aprendizaje de los preescolares (Merino et al., 2014; Quiroga-Lobos et al., 2014), la formación que reciben los profesores de educación básica y las estrategias que utilizan para enseñar ciencias en el aula (Vergara y Cofré, 2008; Cofré et al., 2010), y la relevancia del vocabulario, discurso y alfabetización en la enseñanza de las ciencias (Larraín, 2009; Strasser et al., 2013).

Por otra parte, los resultados en la prueba SIMCE de Ciencias Naturales para estudiantes de cuarto básico muestran que el puntaje promedio nacional se ha mantenido relativamente constante en los años 2007, 2009, 2011 y 2013 (250, 256, 259 y 255 puntos, respectivamente) (MINEDUC, 2014). La tendencia en esta prueba SIMCE se mantiene cuando, para los cuatro años considerados, se analizan los bajos puntajes de estudiantes que exhiben una mayor propensión a la vulnerabilidad y que pertenecen a colegios municipales (235, 239, 245 y 241 puntos, respectivamente), grupos socioeconómicos medio-bajo (231, 237, 243 y 241 puntos) y bajo (226, 228, 234 y 232 puntos). A nivel país, los resultados de la prueba SIMCE de Ciencias Naturales aplicada el año 2013 muestran que aproximadamente un $42 \%$ de los estudiantes se ubicaron en el nivel de aprendizaje insuficiente; es decir, estos estudiantes "no logran demostrar consistentemente que han adquirido los conocimientos y las habilidades más elementales estipulados en el currículum para el período evaluado" (MINEDUC, 2014, p. 6). Si uno considera posibles soluciones a esta problemática, es altamente probable que la implementación de nuevas y adecuadas estrategias de enseñanza de las ciencias durante el nivel preescolar, puedan contribuir a mejorar el desempeño de los estudiantes de cuarto básico en el área de las Ciencias Naturales.

En el país, desde hace unos 13 años se han empezado a realizar importantes innovaciones en la enseñanza del método científico en los niños. A partir de una exitosa metodología indagatoria implementada en Francia y luego en Estados Unidos, en 2002 un 
grupo de educadores e investigadores de la Universidad de Chile y el Ministerio de Educación adaptó el programa "Enseñanza de las Ciencias Basadas en la Indagación" (ECBI) cuyo objetivo es entregar una educación científica de calidad a todos los niños (Devés y Reyes, 2007). El espíritu de este programa es que una ciencia bien enseñada y guiada por profesores capacitados permitirá a los niños obtener una mejor comprensión del mundo natural y material, y experimentar el placer de investigar y descubrir, apropiándose de un pensamiento científico, desarrollando formas de convivencia, generando una comunicación efectiva, fomentando el trabajo en equipo, respeto por las ideas de los otros, y el cuidado por la naturaleza. El foco, en una primera instancia, estuvo puesto principalmente en los cursos de $5^{\circ}$ a $8^{\circ}$ Básico de escuelas municipalizadas, donde se utilizaron módulos de trabajo en ciencias naturales tales como vida y desarrollo de las plantas, sistema nervioso, ecosistemas, sexualidad, y evolución. Ya el año 2007, la cobertura de este programa llegaba aproximadamente a 40 mil niños. El año 2009, se comienza a incorporar gradualmente el nivel de la Educación Parvularia a través de la capacitación en servicio efectuada a sus profesionales, lo cual se traduce en la creación del modulo "del Trigo al Pan".

En la misma línea, el Programa EXPLORA, a través de su iniciativa "Tus Competencias en Ciencias” (TCC) ha fomentado la valoración de la ciencia en el mundo escolar a través de talleres participativos cuyo propósito es ofrecer un espacio para que los niños desarrollen las diferentes habilidades científicas. Esta iniciativa comenzó a operar el año 2007, y su alcance ha llegado a decenas de miles de niños entre $5^{\circ}$ básico y $2^{\circ}$ medio. En el año 2009 se incluyó a la Educación Parvularia y Primer Ciclo de Enseñanza Básica a través del diseño de materiales didácticos (unidades de indagación, experimentación y proyección, material complementario, entre otros) y, puntualmente, en el año 2012 un total de 280 educadoras de párvulos fueron seleccionadas para implementar esta iniciativa en diferentes jardines, escuelas y colegios a lo largo del país.

\section{INDAGACIÓN CIENTÍFICA}

Durante los primeros cinco años de vida de un niño, ocurren la mayoría de los estados cruciales del desarrollo de su cerebro (Kuo et al., 2010). De hecho, en esta etapa, los niños comprenden no solo conceptos científicos abstractos sino que también son capaces de involucrarse en la indagación científica e inferir nuevo conocimiento sobre la base de la experimentación basada en el juego (Gopnik, 2012). Por este motivo, en la actualidad, la mayoría de los educadores concuerdan que la enseñanza de las ciencias debe comenzar en los primeros años de vida (Eshach et al., 2011). Al respecto, es bien conocido que los niños pequeños pueden aprender conceptos y vocabulario científico y habilidades para resolver problemas en ciencias cuando son guiados de manera apropiada y se les provee de una etapa de instrucción (Hong y Diamond, 2012). Es importante aprovechar las 
bases conceptuales y habilidades básicas que poseen los niños a esta edad sobre la ciencia, pues la visión que ellos tienen del mundo que les rodea puede llevarlos a construir ideas erróneas que afectarán la forma en que éstos entienden fenómenos, aprenden conceptos y aplican habilidades científicas (Smolleck y Hershberger, 2011).

Desde el momento en que nacen, los niños son naturalmente inquisitivos y comienzan a hacer ciencia al observar e intentar clasificar su mundo (Martin et al., 2005). Los niños piensan y actúan como científicos cuando efectúan actividades recreativas y exploran su entorno. En particular, hacen ciencia cada día cuando, por ejemplo, en sus juegos observan y descubren las particularidades de su ambiente, generando así un entendimiento ingenuo acerca del mundo en el cual viven (Trundle y Sackes, 2010; Smolleck y Hershberger, 2011). De hecho, son considerados científicos naturales (Brenneman, 2009). Esencialmente, ellos cultivan la indagación, un concepto heurístico que consta de cinco pasos (preguntar, predecir, experimentar, replicar, aplicar) y en el que términos como problema, hipótesis, experimento y evaluación están continuamente interactuando entre sí en pasos cíclicos (Dejonckheere et al., 2010). En este contexto, se ha planteado que futuras investigaciones debieran concentrar los esfuerzos en analizar cómo el círculo de la indagación científica opera en niños entre los tres y seis años de edad (Siry et al., 2012).

Los niños espontáneamente participan en un proceso de indagación científica, maravillándose, formulando preguntas, planificando como responder estas preguntas, documentando sus trabajos, compartiendo sus resultados con otros, y pensando acerca de qué hacer a continuación (Ahsbrook, 2006). Específicamente, ellos están continuamente observando, describiendo los objetos, y cuestionando la ocurrencia de hechos en su entorno. De esta forma, hacen ciencia al identificar, comparar una cosa con otra, y comunicar sus hallazgos (Armga et al., 2002). Para un niño la ciencia es descubrimiento. En el descubrimiento de su mundo, los niños usan los cinco sentidos -ver, escuchar, tocar, probar y oler- para explorar de forma activa su alrededor. Animales como los caracoles, gusanos y orugas, entre otros, fascinan a los niños y constituyen un modelo adecuado para que ellos pongan en práctica sus sentidos y puedan así observar y conversar sobre estos seres vivos durante horas (Plevyak y Arlington, 2012).

La observación corresponde a una actividad mental que no solo involucra las respuestas de los sentidos a un estímulo sino que también las ideas previas y expectativas del sujeto (Harlen, 2007). Si bien la observación ha sido reconocida como una habilidad inicial importante en la enseñanza de la ciencia, el desarrollo y potenciación de esta competencia en la primera infancia ha recibido poca atención (Johnston, 2009). La observación es un prerrequisito clave para el uso exitoso del resto de los pasos del método científico, ya que de algún modo siempre está implícita en actividades como formular preguntas, elaborar hipótesis, interpretar datos y generar nuevas conclusiones científicas 
(Kohlhauf et al., 2011). Dado que desde los cuatro años, los niños son capaces de plantear preguntas e hipótesis, se debe fomentar la práctica de la observación como una competencia básica en ciencia. Es importante consignar que a esta edad, la observación puede ser influenciada por precondiciones del aprendizaje como el interés dominio-específico (los niños observan solo lo que les interesa), las ideas previas, y las habilidades de lenguaje (Johnston, 2009; Kohlhauf et al., 2011; Gopnik, 2012).

Durante la exploración del mundo que los rodea, las observaciones iniciales que los niños realizan, involucran -especialmente- el uso de los sentidos del tacto, audición y visión (Johnston, 2009). En una primera instancia, los niños pequeños tienden a encontrar las características que diferencian a los objetos antes que aquellas que los asemejan; luego, en un paso posterior, ellos se fijan en detalles que les sirven para agrupar y clasificar objetos (o secuencias de eventos), es decir, efectúan una categorización de los objetos según la función que cumplen (Harlen, 2007; Johnston, 2009; Hess, 2010).

El contexto (actividad, ambiente, recursos) en el cual los niños observan fenómenos naturales como, por ejemplo, el comportamiento de los animales, produce efectos positivos sobre el desarrollo del lenguaje, las actitudes e interacciones sociales (Maynard y Waters, 2007; Tompkins y Tunnicliffe, 2007). De esta forma, el uso de objetos o fenómenos científicos motivadores permite a los niños realizar buenas observaciones, las cuales pueden quedar registradas en forma de actuaciones teatrales, escritos, y/o representaciones gráficas (dibujos) (Murray y Valentine-Anand, 2008; Howitt et al., 2011; Stoll et al., 2012).

Los dibujos de los niños son un reflejo de lo que ellos sienten, conocen y/o entienden acerca de un concepto (Chang, 2012). La técnica del dibujo ha sido utilizada en niños, desde los cuatro años, para recoger expresiones acerca de la ocurrencia de eventos, discusiones dentro del grupo de compañeros, interpretaciones acerca de perturbaciones ecológicas, y potenciar el desarrollo de una inteligencia espacial y naturalista (Ergazaki y Andriotou, 2010; Kuo et al., 2010). Además, el dibujo tiene la ventaja de superar las barreras lingüísticas y permitir la comparación entre grupos con diferente habilidad de lenguaje (Chambers, 1983). En relación con la percepción ambiental, el dibujo permite efectuar un análisis de contenido sobre símbolos e imágenes, lo cual permite conocer las concepciones que tienen los niños acerca del funcionamiento de la ciencia (Barraza, 1999; Chang, 2012). Un supuesto importante al trabajar con este método es que existe un paralelismo entre el desarrollo cognitivo del niño (que va desde lo sensorial hasta las operaciones concretas y formales) y el desarrollo de sus dibujos (Piaget, 1969; Piaget y Inhelder, 1971). Los niños de tres a cuatro años se encuentran en la etapa del realismo simbólico, es decir, comienzan a representar y relacionar detalles en sus dibujos; mientras que entre los cinco y siete años se encuentran en la etapa del realismo intelectual, es decir, sus dibujos contienen elementos que ellos saben que existen a pesar de no haberlos visto, siendo esto un fiel reflejo del impacto del conocimiento. Hasta la fecha, existe una escasa 
integración entre los dibujos realizados por los niños y sus logros conceptuales en ciencia (Chang, 2012).

De acuerdo a Harlen (2007) "preguntar es una forma poderosa de reclamar una respuesta de alguien. Los niños preguntan en clase para ampliar sus conocimientos y... por curiosidad” (p. 101). Cuando los niños formulan preguntas, se familiarizan con habilidades básicas de la ciencia como observar más cuidadosamente, aprender nombres de objetos y procesos, y comparar cosas (Armga et al., 2002). Cuando formulan preguntas centradas en el qué, cómo, y por qué, los niños activan procesos de pensamiento y aprendizaje (Armga et al., 2002; Harlen, 2007). Así por ejemplo, el proceso que involucra encontrar la solución a un problema, puede verse potenciado cuando los niños escuchan y aprenden de las preguntas formuladas por sus compañeros de clase (Johnston, 2009; Gopnik, 2012; Mills et al., 2012). El estímulo y apoyo para que los niños practiquen y refinen la formulación de preguntas de cualquier tipo, servirá para retroalimentar otras habilidades científicas básicas como observar, identificar, comparar, clasificar y comunicar (Armga et al., 2002; Harlen, 2007; Ashbrook, 2011). De esta forma, las preguntas constituyen el medio para que el niño conecte distintas experiencias, facilitando así la construcción de su propia imagen del mundo (Harlen, 2007).

Las preguntas que efectúan los niños pueden ser de cuatro tipos: i) comentarios o expresión de interés, una manera de decir; ii) informativas, que recaban información directa; iii) requieren de una respuesta compleja que trasciende la capacidad de comprensión de los niños, es decir, necesitan de una explicación, los complicados ¿por qué?; y iv) deben ser investigadas por los niños, es decir, se generan en el contexto de una actividad, y corresponden a cuestionamientos comprobables (Harlen, 2007).

Junto con las preguntas que surgen al interior del aula, se debe fomentar que los niños realicen actividades fuera de la sala de clases para que así sean capaces de cuestionarse sobre el entorno exterior, y de este modo relacionen los fenómenos reales con los contenidos abordados en las actividades de clase (Harlen, 2007; Roach, 2010; Daza et al., 2011; Feinstein et al., 2013). Confrontar a los niños a observar los seres vivos de su entorno, es una estrategia que les provee de herramientas conceptuales que facilitan la formulación de preguntas tanto en la situación actual como en futuras actividades (Eshach et al., 2011). En particular, las preguntas que surgen en relación con la observación de animales, pueden ser agrupadas -de menor a mayor complejidad- en tres categorías: forma física del animal, ambiente en que vive el animal, y conducta del animal (Braund, 1998; Shepardson, 2002; Tunnicliffe et al., 2008; Eshach et al., 2011). 


\section{LA IMPORTANCIA DE REALIZAR INVESTIGACIÓN EN CIENCIAS NATURALES EN EL NIVEL \\ PREESCOLAR: LA BIOFILIA COMO UNA OPORTUNIDAD}

\section{UN PROYECTO DE INVESTIGACIÓN CENTRADO EN LA BIOFILIA}

Desde comienzos de 2014 estamos desarrollando, junto a las co-investigadoras Mónica Manhey y Carolina Navarro, el proyecto FONDECYT Regular No 1140812 "Observar y preguntar: evolución de habilidades de pensamiento científico durante el primer y segundo nivel de transición”. Esta investigación focaliza la atención en el Ámbito: Relación con el Medio Natural y Cultural, Núcleo: Seres Vivos y su Entorno, Eje: Descubrimiento del Medio Natural; dado que al revisar las Bases Curriculares y los Programas Pedagógicos del primer y segundo nivel de transición (MINEDUC, 2005, 2008), es posible destacar los siguientes aprendizajes en que los niños debieran desarrollar sus capacidades para observar y preguntar: i) comprender cómo la alimentación y locomoción de los seres vivos se relaciona con su hábitat, ii) identificar las necesidades, características y cambios que ocurren en los procesos de crecimiento y desarrollo de los seres vivos, y iii) reconocer, apreciar y representar plantas, animales, y fenómenos naturales presentes en el entorno del niño.

Este tipo de aprendizajes es coincidente con los intereses que manifiestan los niños desde temprana edad- por establecer contacto y empatía con la naturaleza. Específicamente, a esta edad los seres humanos establecen una afiliación innata hacia otros organismos vivos, en particular, curiosidad por conocer las conductas de locomoción, alimentación y selección de hábitats, entre otras, que exhiben los animales, lo cual se conoce como el concepto de biofilia (Wilson, 1984; Kellert y Wilson, 1993). Este comportamiento que manifiestan los niños, como todo carácter del fenotipo de un individuo, tiene un componente genético y otro ambiental (aprendizaje) (Barraza, 1998). Es en este último aspecto donde las estrategias de educación deben potenciar las habilidades de pensamiento científico que los niños pueden llegar a desarrollar. De hecho, si bien los niños a esta edad son capaces de reconocer algunos elementos de la flora y fauna de su entorno, existe una tendencia de parte de ellos a considerar la naturaleza como una entidad estática y libre de intervención humana. Este tipo de preconcepciones son importantes de cambiar, para así aspirar a que los niños construyan una imagen adecuada del mundo que los rodea y de los problemas ambientales a los que se verán confrontados en su vida adulta, fomentando en ellos actitudes e intereses sobre cómo se investiga y se aprende en el ámbito de las ciencias.

Esencialmente, el objetivo general de este estudio es: Comprender la evolución de las observaciones y preguntas que efectúan los niños durante el Primer y Segundo Nivel de Transición, cuando participan en experiencias de aprendizaje en las que se relacionan con otros seres vivos. Siendo los objetivos específicos: i) Describir los cambios temporales en los tipos de observaciones y preguntas que los niños son capaces de realizar durante la educación parvularia. ii) Entender las conexiones entre el espacio físico en que se desarrolla la actividad pedagógica (dentro o fuera del aula) y el tipo de observaciones y preguntas que 
los niños son capaces de realizar. iii) Explorar la vinculación entre el contexto del colegio (dependencia administrativa, nivel socioeconómico) y el tipo de observaciones y preguntas que los niños son capaces de realizar. iv) Identificar el vocabulario con el que ingresan los niños al Primer Nivel de Transición. v) Conocer el tipo de observaciones y preguntas sobre animales que inicialmente los apoderados y las educadoras de párvulos promueven en sus hijos y alumnos, respectivamente.

Específicamente, se pretende evaluar las siguientes dos hipótesis de trabajo. Hipótesis de la observación: Con el paso del tiempo, se producirá en los niños un cambio desde observaciones focalizadas en características simples (similitudes y/o diferencias de distintos animales) a otras centradas en detalles complejos (secuencia que sigue la conducta de un animal), proceso que diferirá según la condición inicial del niño y el contexto del colegio al cual asiste. Hipótesis de la pregunta: Con el paso del tiempo, se producirá en los niños un cambio desde preguntas centradas en el interés (comentarios, expresiones y/o maneras de decir) a otras que requieren de investigación (cuestionamientos comprobables en el contexto de una actividad práctica), proceso que diferirá según la condición inicial del niño y el contexto del colegio al cual asiste.

La investigación corresponde a un estudio de tipo exploratorio-descriptivo, el diseño es no-experimental de tipo longitudinal y -específicamente- corresponde a un estudio de panel. El trabajo está siendo abordado mediante un enfoque mixto, es decir, se utilizan métodos cualitativos y cuantitativos con el objetivo de obtener una mayor riqueza de información, lograr un mejor conocimiento, y/o abordar la problemática en mayor profundidad. Respecto a los centros educativos (muestras seleccionadas de manera noprobabilística o dirigida), se está trabajando con un total de seis colegios pertenecientes a la Región Metropolitana (estudios de caso de tipo colectivo): dos corresponden a la dependencia administrativa municipal (Escuela Cadete Arturo Prat, Colegio Villa Macul), dos son de tipo particular subvencionado (Colegio Libertador Simón Bolívar, Colegio Emmanuel High School), y dos son particulares pagados (Colegio Rubén Darío, Liceo Experimental Manuel de Salas). En relación con los sujetos de estudio (unidad de análisis), se trabajará con una cohorte de niños que en marzo de 2014 ingresaron al primer nivel de transición (pre-kínder), la cual cursará durante el año 2015 el segundo nivel de transición (kínder). Es decir, a lo largo de estos dos años se documentará para cada niño (evaluación idiográfica del aprendizaje) el tipo de observaciones y preguntas que son capaces de realizar acerca de diferentes animales (invertebrados y vertebrados) en actividades realizadas al interior del aula, las cuales quedarán plasmadas mediante la confección de dibujos. En el año 2016, y con el fin de evaluar la efectividad de experiencias de aprendizaje efectuadas en el exterior del aula sobre la calidad de las observaciones y preguntas, se trabajará con dos cohortes distintas de niños; una que se encuentre cursando el primer nivel de transición, y la otra que esté en el segundo nivel de transición. 


\section{LA IMPORTANCIA DE REALIZAR INVESTIGACIÓN EN CIENCIAS NATURALES EN EL NIVEL

A continuación, se detallan brevemente las actividades que hemos realizado durante el año escolar 2014. Durante el primer semestre, en una primera instancia se procedió a conseguir los consentimientos informados de las autoridades institucionales, educadoras de párvulos y los representantes de los estudiantes (en este último caso, para asegurar que cada niño participe de manera voluntaria en el estudio). Además, se aplicó a los apoderados un cuestionario mixto que contenía tanto preguntas cerradas como abiertas, con el objetivo de descubrir formas de conductas y determinar el tipo de relaciones que ellos establecen con sus hijos respecto a observaciones y preguntas sobre los seres vivos. La conveniencia de efectuar cuestionarios que planteen situaciones de la vida cotidiana, dice relación con la posibilidad de recopilar manifestaciones de actitudes, puntos de vista y/o valoraciones del entorno familiar del niño, aspectos que son claves en los resultados que se alcancen en el proceso de aprendizaje durante la etapa preescolar. También se procedió a evaluar el nivel de vocabulario receptivo de los niños que ingresan al primer nivel de transición mediante el Test de Vocabulario en Imágenes Peabody (TVIP). Este test consiste en confrontar al niño a láminas que contienen cuatro imágenes y solicitarle que luego de escuchar una palabra (estímulo) identifique la imagen asociada; este procedimiento entrega información sobre el desarrollo del lenguaje y proyección del habla que tienen los preescolares cuya lengua materna es el idioma español, y constituye una medida de la variabilidad existente en los individuos evaluados (Strasser et al., 2010). Por último, en cada uno de los establecimientos educacionales se procedió, mediante la técnica de la observación noparticipante, a registrar el tipo de enseñanza (experiencias de aprendizaje) que las educadoras de párvulos fueron capaces de transmitir a los niños a través de sus intervenciones, explicaciones y/o interacciones en el Ámbito Relación con el Medio Natural y Cultural.

Durante el segundo semestre de 2014, se implementaron experiencias de aprendizaje consistentes en mostrar a los niños diferentes tipos de vertebrados e invertebrados terrestres representantes de nuestra fauna nativa. En particular, a comienzos de semestre los modelos animales que se utilizaron fueron el ratón cola de pincel o degú (Octodon degus) y la araña pollito (Grammostola rosea), y a fines de semestre la laucha orejuda de Darwin (Phyllotis darwini) y el palote (Bacunculus granulicollis). Luego de finalizada la exhibición del animal al curso, se solicitó a los niños que representaran en un dibujo el ejemplar que fue observado por ellos. Para el desarrollo de esta actividad, se proveyó a cada niño de hojas de block de dibujo y lápices de cera a colores. Al finalizar sus dibujos, y tras un breve descanso, cada uno de los niños fue entrevistado -cuando existieron las condiciones- en una sala habilitada especialmente para que la conversación se desarrollara de manera natural y en un ambiente tranquilo y familiar. Se generó una entrevista semi-estructurada muy sencilla que contenía dos preguntas centrales tendientes a obtener información acerca de las observaciones e inquietudes que a los niños les surgieron luego de la actividad. En todo momento, el niño tuvo a disposición su dibujo y de esta manera fue posible conversar con él sobre el significado de las características y detalles de 
cada elemento y símbolo registrado en la representación gráfica. Tanto en la actividad grupal de confección de los dibujos como en las conversaciones personales, se efectuaron grabaciones de audio con el fin de no perder detalles sobre otras variables que pudieran ser de interés (número de alumnos por clase opinando, diferencias de género, sentimientos, actitudes y motivaciones, entre otros; Hardman et al., 2005).

En la actualidad, se está terminando de transcribir las entrevistas de los niños y se está comenzando a analizar sus dibujos. Respecto a estos instrumentos, los análisis permitirán efectuar distinciones acerca del grado de complejidad de las observaciones efectuadas por los niños. Así por ejemplo, algunos elementos o variables reconocidas en los dibujos serán agrupadas y clasificadas (forma, tamaño, color, movimiento y conducta de los animales) para obtener así información acerca de su frecuencia y/o la naturaleza de su significado. Además de los cuatro tipos de preguntas generales (interés, informativas, explicativas, investigativas) y los tres grupos de preguntas enfocadas en los animales (forma, hábitat y comportamiento), se procederá a categorizar las preguntas según éstas apunten a: la temporalidad (¿cuándo?), la espacialidad (¿dónde?), la cantidad (¿cuánto?), los objetos (¿qué?, ¿quién?, ¿cuál?), y los mecanismos (¿cómo?, ¿por qué?).

Se espera que los resultados obtenidos en este proyecto, permitan dilucidar interrogantes como: ¿los niños provenientes de diferentes tipos de establecimientos comienzan en igualdad de condiciones su formación preescolar como científicos?, ¿los cambios que experimentan los niños desde observaciones simples a complejas y/o desde preguntas centradas en el interés a otras que requieren investigar, se producen a una misma tasa en los diferentes tipos de colegios?, ¿ al final de la educación parvularia los niños que asisten a distintos tipos de establecimientos alcanzan los mismos logros de aprendizaje en el Ámbito Relación con el Medio Natural y Cultural?

Finalmente, es importante mencionar que la información existente sobre la temática de ciencia y educación parvularia que circula al interior de casas de estudio universitarias, centros de investigación e instituciones públicas, debiera ser publicada en revistas con comité editorial y revisión de pares para que de esta forma quede a disposición de la comunidad científica y/o público en general. Además, debiera existir interés de parte de las autoridades que imparten las carreras de educación parvularia para que desde su ingreso (ponderación de la prueba de ciencia) y durante su formación inicial (asignaturas, prácticas, unidades de investigación, seminarios de título) nuestras futuras educadoras de párvulo se vayan familiarizando con el quehacer de la investigación científica dentro y fuera del aula. 


\section{AGRADECIMIENTOS}

Agradezco al Director y Editora de la Revista Enfoques Educacionales la invitación a escribir este artículo. La investigación mencionada en el texto es financiada por el Proyecto FONDECYT Regular No 1140812, CONICYT, Chile.

\section{REFERENCIAS BIBLIOGRÁFICAS}

Armga, C., S. Dillon, M. Jamsek, P.D. Jolley, E.L. Morgan, D. Peyton \& H. Speranza, 2002 Tips for helping children do science. Texas Child Care 26: 2-7.

Ashbrook, P., 2006 Mixing and making changes. Science and Children 43: 28-31.

Ashbrook, P., 2011 Ongoing inquiry. Science and Children 48: 22-23.

Barraza, L., 1998 Conservación y medio ambiente para niños menores de 5 años. Especies 7: 19-23.

Barraza, L., 1999 Children drawings about the environment. Environmental Education Research 5: 49-66.

Braund, M., 1998 Trends in children's concepts of vertebrate and invertebrate. Journal of Biological Education 32: 112-118.

Brenneman, K., 2009 Let’s find out! Preschoolers as scientific explorers. Young Children 64: 54-60.

Chambers, D.W., 1983 Stereotypic images of the scientist: The draw-a-scientist test. Science Education 67: 255-265.

Chang, N., 2012 What are the roles that children's drawings play in inquiry of science concepts? Early Child Development and Care 182: 621-637.

Cofré, H., J. Camacho, A. Galaz, J. Jiménez, D. Santibáñez \& C. Vergara, 2010 La educación científica en Chile: Debilidades de la enseñanza y futuros desafíos de la educación de profesores de ciencia. Estudios Pedagógicos 36: 289-303. 
Daza, S. y M. Quintanilla, 2011 La enseñanza de las ciencias naturales en las primeras edades: su contribución a la promoción de competencias de pensamiento científico. Santander, Colombia: Barrancabermeja.

Daza, S., M. Quintanilla, J. Arrieta \& E. Benedetti, 2011 El preguntario de los niños, para cada genuina pregunta existe una sabia respuesta. En La enseñanza de las ciencias naturales en las primeras edades: Su contribución a la promoción de competencias de pensamiento científico (Daza S \& M Quintanilla, editores). Santander, Colombia: Barrancabermeja. Pp. 82-110.

Dejonckheere, P.J.N., K. Van de Keere \& N. Mestdagh, 2010 Training the scientific thinking circle in pre- and primary school children. The Journal of Educational Research 103: 1-16.

Devés, R. y P. Reyes, 2007 Principios y estrategias del programa de educación en ciencia basada en la indagación (ECBI). Pensamiento Educativo 41: 115-131.

Ergazaki, M. y E. Andrioton, 2010 From "forest fires" and "hunting" to disturbing "habitats" and "food chains": Do young children come up with any ecological interpretations of human interventions within a forest? Research in Science Education 40: 187-201.

Eshach, H., Y. Dor-Ziderman \& Y. Arbel, 2011 Scaffolding the “scaffolding” metaphor: From inspiration to a practical tool for kindergarten teachers. Journal of Science Education and Technology 20: 550-565.

Feinstein, N.W., S. Allen \& E. Jenkins, 2013 Outside the pipeline: Reimagining science education for nonscientists. Science 340: 314-317.

Gopnik, A., 2012 Scientific thinking in young children: Theorethical advances, empirical research, and policy implications. Science 337: 1623-1627.

Hardman, F., F. Smith \& K. Wall, 2005 Teacher-pupil dialogue with pupils with special educational needs in the National Literacy Strategy. Educational Review 57: 299-316.

Harlen, W., 2007 Enseñanza y aprendizaje de las ciencias. Madrid, España: Ediciones Morata. 
Hepp, P., M.E. Merino, M.V. Barriga \& A. Huircapán, 2013 Tecnología robótica en contextos escolares vulnerables con estudiantes de la etnia Mapuche. Estudios Pedagógicos 39: 75-84.

Hess, K.K., 2010 Using learning progressions to monitor progress across grades. Science and Children 47: 57-61.

Hong, S.-Y. y K.E. Diamond, 2012 Two approaches to teaching young children science concepts, vocabulary, and scientific problem-solving skills. Early Childhood Research Quarterly 27: 295-305.

Howitt, C., S. Lewis \& E. Upson, 2011 "It's a mystery!” A case study of implementing forensic science in preschool as scientific inquiry. Australasian Journal of Early Childhood 36: 45-55.

Johnston, J.S., 2009 What does the skill of observation look like in young children? International Journal of Science Education 31: 2511-2525.

Kellert, S.T. y E.O. Wilson, 1993 The biophilia hypothesis. Island Press, Washington, U.S.A.

Kohlhauf, L., U. Rutke \& B. Neuhaus, 2011 Influence of previous knowledge, language skills and domain-specific interest on observation competency. Journal of Science Education and Technology 20: 667-678.

Kuo, Ch.-Ch., J. Marker, F.-L. Su \& Ch. Hu, 2010 Identifying young gifted children and cultivating problem solving abilities and multiple intelligences. Learning and Individual Differences 20: 365-379.

Larraín, A., 2009 El rol de la argumentación en la alfabetización científica. Estudios Públicos 116: 167-193.

López de Maturana, D., 2010 El juego como manifestación cuántica: una aproximación a la epistemología infantil. Polis 25: 243-254.

Martin, D.J., J.S. Raynice \& E. Schmidt, 2005 Process-oriented-inquiry a constructivist approach to early childhood science education: Teaching teachers to do science. Journal of Elementary Science Education 17: 13-26. 
Maynard, T. y J. Waters, 2007 Learning in the outdoor environment: A missed opportunity? Early Years 27: 255-265.

Merino, C., C. Olivares, A. Navarro, K. Avalos \& M. Quiroga, 2014 Tus competencias en ciencias en educación parvularia: ¿nuestra cocina es un laboratorio de química? Educación Química 25: 229-239.

Mills, C.M., J.H. Danovitch, M.G. Grant \& F.B. Elashi, 2012 Little pitchers use their big ears: Preschoolers solve problem by listening to others ask questions. Child Development 83: 568-580.

MINEDUC, 2005 Bases Curriculares de la Educación Parvularia.

MINEDUC, 2008 Programas Pedagógicos Primer y Segundo Nivel de Transición.

MINEDUC, 2014 SIMCE 2013, Síntesis de Resultados, 4 Educación Básica.

Murray, M. y L. Valentine-Anand, 2008 Dinosaur extinction, early childhood style. Science and Children 46: 36-39.

Piaget, J., 1969 The child's conception of the world. Routledge and Kegan Paul Ltd., London.

Piaget, J. y B. Inhelder, 1971 Mental imagery in the child. Basic Books, New York.

Plevyak, L. y R. Arlington, 2012 Kindergarteners, fish, and worms ... Oh my! Science Children 50: 54-59.

Quiroga-Lobos, M.E., E. Arredondo-González, D. Cafena \& C. Merino-Rubilar, 2014 Desarrollo de competencias científicas en las primeras edades: el Explora Conicyt de Chile. Educación y Educadores 17: 237-253.

Roach, J., 2010 Sowing the seeds of science understanding in the early years. Primary Science 111: 16-19.

Sánchez, A.P., 2014 La emergencia metafórica y propensión a aprender de preescolares en Chile y Brasil. Polis 37: 233-249.

Shepardson, D.P., 2002 Bugs, butterflies, and spiders: Children's understandings about insects. International Journal of Science Education 24: 627-643. 
Siry, C., G. Ziegler \& C. Marx, 2012 "Doing science" through discourse-in-interaction: Young children's science investigations at the early childhood level. Science Education 96: 311-336.

Smolleck, L. y V. Hershberger, 2011 Playing with science: an investigation of young children's science conceptions and misconceptions. Current Issues in Education 14: 431.

Stoll, J., A. Hamilton, E. Oxley, A.M. Eastman \& R. Brent, 2012 Young thinkers in motion: Problem solving and physics in preschool. Young Children 67: 20-26.

Strasser, K., A. Larraín, S. López de Lérida \& M.R. Lissi, 2010 La comprensión narrativa en edad preescolar: Un instrumento para su medición. Psykhe 19: 75-87.

Strasser, K., F. Del Río \& A. Larraín, 2013 Profundidad y amplitud de vocabulario: ¿Cuál es su rol en la comprensión de historias en la edad pre-escolar? Estudios de Psicología 34: 221-225.

Tompkins, S.P. y S.D. Tunnicliffe, 2007 Nature tables: Stimulating children's interest in natural objects. Journal of Biological Education 41: 150-155.

Trundle, K.C. y M. Sackes, 2010 Look! It is going to rain. Science and Children 47:2931.

Tunnicliffe, S.D., S. Gatt, C. Agius \& S.A. Pizzuto, 2008 Animals in the lives of young Maltese children. Eurasia Journal of Mathematics, Science \& Technology Education 4: 215-221.

Vergara, C. y H. Cofré, 2008 La enseñanza de las Ciencias Naturales en la Educación Básica chilena: Un camino por recorrer. Revista Foro Educacional 14: 85-104.

Wilson, E.O., 1984 Biophilia: the human bond with other species. Harvard University Press, Harveard, U.S.A. 